\title{
Construcción de un entorno conceptual alrededor del tema del tránsito urbano
}

\section{Construction of a conceptual environment around the theme of urban transit}

Nancy Mariela Casadiego-Ruiz'

Recibido: octubre 13 de 2016 Aceptado: diciembre 16 de 2016

\section{Resumen}

Puesto que la transformación conceptual sobre conocimiento y ciencia del siglo XX trajo un cambio en la racionalidad científica prevalente hasta el Siglo XIX con un nuevo paradigma epistemológico que cuestiona el modelo científico positivista, se intentan conciliar puntos de vista sobre el tema de la interacción de los actores del tránsito urbano desde un enfoque que, sin agotar el espectro, va más allá de la consideración de la movilidad desde aspectos netamente tempo-espaciales. Se aborda el tema desde la filosofía, la psicología y la sociología en relación con el uso del espacio para interpretar cambios de comportamiento en relación con tendencias de crecimiento exagerado del tránsito y los conflictos que se generan en el espacio público. Lo anterior, con la intención de suscitar una reflexión enriquecedora de diferentes puntos de vista para quienes se dedican a la formación en la Ingeniería del Tránsito, con una posible exploración interdisciplinar que contribuya a la creación de formas didácticas novedosas, destinadas a lograr cambios en la forma de ver los actores del tránsito urbano para la redefinición de normas, la promoción, aplicación o investigación acerca de la eficacia de las acciones orientadas al control conductual en la vía urbana.

Palabras clave: comportamiento, educación, norma, tránsito urbano.

\begin{abstract}
Considering that the mindset transformation in regards to the knowledge and science during 20th-century, a change in scientific rationality that prevailed until the 19th Century was brought as a new epistemological paradigm questioning the positivist scientific model. The present article aims to reconcile points of view regarding the interaction among urban transit actors, using an approach that goes beyond contemplating mobility from the temporal-spatial aspect only. This article covers topics from philosophy, psychology and sociology in relation to the use of space in order to interpret behaviour changes linked to overgrowth tendencies in transit and conflicts generated in public space. The foregoing aims to promote an enriching reflection that considers different points of view for those who work in the education branch of Traffic Engineering, with a potentially interdisciplinary exploration that contributes to the creation of new didactic resources intended for achieving changes in the way of seeing the transit urban actors in pursuance of redefining norms and the promotion, application or research regarding the efficacy of actions oriented to control behaviour in urban areas.
\end{abstract}

Keywords: behaviour, education, norm, urban transit. 


\section{Introducción}

La transformación de conceptos sobre el conocimiento y la ciencia del siglo XX inician con un cambio de enfoque de la racionalidad científica prevalente hasta el Siglo XIX, dando paso a un nuevo paradigma epistemológico que cuestiona el modelo científico positivista. Este movimiento fue iniciado por los psicólogos de la Gestalt (Arnoletto, 2007), y continuó en la tercera década del siglo XX con los biólogos y los filósofos de la ciencia (Popper, 1987), quienes paulatinamente han ido configurando un cambio de enfoque sobre el conocimiento con múltiples aportes de insatisfacción con la forma de racionalidad lineal, unidireccional, cartesiana; lo anterior para llegar a establecer poco a poco la necesidad de reemplazar un modelo axiomático de pensar, razonar y demostrar, basado en el ideal puro de lógica formal o lógica matemática, con el aporte de un nuevo discurso que da cabida a una más auténtica, compleja y empírica mirada a la realidad del mundo de la cotidianidad con el que interactuamos. Un mundo en el que hay inconsistencias, incoherencias lógicas y hasta contradicciones conceptuales, pero que en últimas enriquece los intentos de su interpretación (Migueles, 2006).

En concordancia con lo anterior e inspirado en el enfoque interdisciplinario para el estudio, generación y validación del conocimiento de las ciencias (Iranzo, 2005), en el presente escrito se intentan canalizar diversas miradas relacionadas con el tema del espacio, el tiempo y la historia en cuanto la conformación de la ciudad como territorio a partir de su relación como objeto geográfico social y natural y los procesos socio-económicos, institucionales, religiosos, políticos, ideológicos y científico-tecnológicos que constituyen sistemas de acciones y flujos de información que como «haz de fuerzas sociales» se ejercen en un lugar conformando la localización como espacio y, a partir de este, la consideración que implican la movilidad y la masificación propios de la ciudad actual, con el propósito de dilucidar las fuertes presiones socio económicas que se ejercen sobre el individuo, y la sinergia que le inhibe alejarse del influjo de la transformación social (Camargo, 2003).

Se hace énfasis en el comportamiento del individuo como usuario actor del espacio urbano, específicamente en el ámbito de la utilización de la vía de circulación. Al incursionar en este tema se espera llegar a una mejor comprensión de las interacciones entre los actores y efectos del fenómeno urbano y a partir de ello, acentuar el enfoque del tema de la interacción entre el ser humano y el entorno vial, o necesidad de moverse en espacios de uso común o público. Esta situación se agudiza cada día debido a la exagerada proliferación de vehículos motorizados, como otro actor importante del tránsito que influye en la expansión del territorio de la ciudad con el incremento de oportunidades, pero que a la vez, ha sido fuente de múltiples efectos, no siempre deseables en los campos económico, social, y en especial, el cultural. Aspectos que sin duda deben entrar en la temática y formación de quienes se dedican a estudiar, diseñar, operar y controlar el tránsito.

\section{Marco Teórico}

\subsection{El espacio vital}

En primer lugar, desde el punto de vista biológico y de acuerdo con Lewin (1964), el concepto de espacio vital desde la Teoría de Campo hace referencia al conjunto de todo aquello que puede afectar al individuo, sea que pertenezca a su espacio físico o no. La necesidad de un lugar para los seres vivos significa algo connatural y reconocible como territorio delimitado de diversas maneras (Bollnow., \& d'Ors, 1969). En las especies vegetales, la dependencia de lugar supone ciertas condiciones que favorecen los procesos de nacimiento y crecimiento a partir de una ubicación estable, la cual puede ser resuelta a través de condiciones favorables para el aprovechamiento de recursos del suelo, humedad, luz y aire. En cuanto a los animales, sus oportunidades de movilización, su percepción y necesidad de territorio se expanden conforme al entorno, como fuente de supervivencia de la espe- 
cie (Hall, 2003) y además, ciertas modalidades que dependerían del grado de organización de convivencia y cooperación de protección conforme a un conjunto de comportamientos como grupo, manada o rebaño (Páez, \& Blanco, 2006).

El ser humano priorizó las necesidades del grupo como tal, percatándose de los beneficios de la cooperación a mediano plazo. En este proceso, pasó del nomadismo o búsqueda dispersa no determinada, a una trashumancia alrededor de un pastoreo móvil regular, relacionado con la mayor seguridad del conocimiento de la productividad de la tierra dentro de un territorio conocido, que lo llevó al establecimiento de asentamientos estacionales fijos, que darían origen a la definición de núcleos principales de los que proviene la aldea como espacio geográfico concreto, en forma y contenido.

Por consiguiente, las primeras civilizaciones de la historia debieron nacer y desarrollarse alrededor de espacios que reunían condiciones propicias para el asentamiento humano: terreno fértil casi siempre cercano a un río o gran masa de agua dulce. Mesopotamia, el territorio regado estratégicamente por el Tigris y el Éufrates, llevó a la plenitud de grandes pueblos 40 siglos A.C. Allí la estabilidad territorial hizo posible la escritura cuneiforme, un gran paso que los nómadas no habrían podido lograr. Por su parte, el Valle del Nilo, muestra vestigios de ocupación humana desde el paleolítico; aunque su territorio presenta escasa pluviosidad, dos crecidas anuales del río garantizaron la fertilidad ribereña dando lugar a otra gran civilización reconocida de todos los tiempos. Allí Egipto, el pueblo de la tierra negra cultivable, creció, dando paso a un gran incremento de su población, su monumentalidad y la escritura jeroglífica. Por su parte, el río Ganges fue destino de todas las invasiones en la antigua India.

En lo que ahora es Europa, la movilidad que ofrecen el mar y el viento del norte, hace mil años empujó los navíos vikingos, hombres de acción, aventureros, piratas y comerciantes del norte, no sujetos a autoridad alguna y sin una patria por la cual morir. De otro lado, desde las estepas del noreste, a la caída del Imperio Romano-Germáni$\mathrm{co}$, dividida tras siglos de migraciones tribales, se atomizó, quedando vulnerable y dando paso al oscurantismo conocido como Edad Media, donde la necesidad de espacios seguros perfila polos de atracción urbana con creciente necesidad de mano de obra, que da origen al "burgo" o congregación alrededor de un poder local.

En resumen, el instinto ancestral del homínido en busca de oportunidades de supervivencia, lo lleva a la premonición de representaciones estimulantes de lo que será al final de su aventura, transformando el espíritu nómada en intercambios de comercio y guerreros (Sanfeliu., \& Varona, 2010). Sin embargo hablar de dichas migraciones en un sentido actual más restringido, solo llegará a tener sentido bajo un principio de propiedad privada o sistema apropiación de bienes, entre ellos, del espacio urbano, donde permanecen los individuos, como rasgo distintivo del hombre de la edad moderna (Wirth, 2005), generando compromisos con el uso del espacio físico. Es la apropiación de un territorio vital habitado y modificado por el hombre, que a su vez ejerce su influencia en quienes lo modifican, explicando y haciendo parte del comportamiento de sus habitantes (Jirón, \& Iturra, 2015).

\subsection{El espacio social}

Son formaciones socio-espaciales que interactúan en las diversas dimensiones de vida del hombre como grupo en lo económico, político, ético, ideológico, religioso, estético, tecnológico y espacial; Instancias que se expresan tanto en la continuidad como en la discontinuidad histórica (Tomadoni, 2007).

Al individuo no le basta con solo disponer de un espacio vital. Le es necesario un espacio de interacciones o espacio relacional; es el "donde" del proceso de ingresar, interactuar y ser aceptado en el grupo, le implica comunicar e interpretar necesariamente la interiorización de pautas culturales, 
narraciones, costumbres y normas; es algo que solo es posible si comparte un lenguaje gestual, oral, gráfico, o escrito, parte de lo que Bourdieu (2005) denomina "Capital Cultural", el cual implica la existencia de una compleja red de relaciones que nacen del entorno familiar inmediato y se van expandiendo al compartir gustos, necesidades, etc., con otros grupos semejantes que se identifican por sus intereses comunes, ocupaciones.

Estas relaciones con frecuencia son mediadas por campos de actividad común: por ejemplo, el académico, en el cual dichas relaciones se basan en la posesión de un capital cultural. Aquí la comunicación se convierte en un medio por el que no solo se aprende a ser miembro de una sociedad particular en el sentido más adaptativo del término, sino que se apropia de la misma y se individualiza en ella, concretando de forma particular y única un entorno social que le ha precedido y del que necesita para poder construirse, ubicarse y ser aceptado a través de relaciones que conforman en conjunto lo que se denomina relaciones sociales; es decir, una sociedad con un determinado grado de desarrollo histórico, una sociedad de carácter peculiar y distintivo.

Surge entonces la noción del espacio físico como escenario y parte de las relaciones entre semejantes, que da origen a la "polis" como producto del crecimiento que proviene de la relación social, donde además de establecer un espacio fijo, contemplaría además un espacio semifijo como extensión del espacio alrededor del cuerpo, cuya amplitud puede variar en función de la cultura en la que se insertan los individuos debido a que cada comunidad social estructura un espacio físico de acuerdo con sus patrones culturales prevalentes. Es un espacio que según Hall (1981) puede definirse a través de un conjunto de hechos y circunstancias que determinan el comportamiento de un sujeto en un momento dado. Este espacio contiene al individuo mismo como ser físico y a su vez a los objetivos que él busca, así como a los factores negativos que trata de eludir; es algo así como una "zona de despeje" en la que se tienen en cuenta las barreras o normas que restringen y limitan sus movimientos, los senderos por los que debe seguir para lograr alcanzar aquello que desea.

Este espacio, al fijar fronteras, da lugar a la delimitación de niveles de espacio entre individuos que permiten establecer distancias subjetivas alrededor de la persona y que en sí se convierten en elemento de comunicación interpersonal, proxémica; distancias o semiesferas de diferentes radios de acción, que incluso pueden ser medidos físicamente, los cuales determinarían los tipos de espacio que deben ser reconocidos y por ende, respetados normalmente entre los individuos en su interactuar. Sin embargo, el espacio social, al igual que el espacio fijo, pueden ser invadidos ocasionalmente por quien no comparte o no ha interiorizado ciertos valores de respeto o límites subjetivos, por ejemplo, al invadir un puesto en una fila de espera, o cuando aún sin invadir el espacio físico, el otro traspasa sus límites de espacio con solo una mirada persistente, al observar fijamente a otra persona durante un intervalo que supera los estándares normales, aunque no invada los límites de la proxemia.

\subsection{El espacio público}

Desde la filosofía, la distinción entre lo íntimo y lo público es objeto de discusión ya desde los clásicos atenienses; Platón en La República y Aristóteles en La Política, quienes reconocen que al concepto de espacio público solo se llega a partir del momento en que el individuo supera su noción de espacio vital privado. Dialécticamente, luego que el individuo ha satisfecho sus necesidades e intereses particulares, está en capacidad de trascender su radio de acción individual o de su grupo familiar, e interactuar con otros, logro al cual solo llegará cuando pueda colectivamente institucionalizar un espacio con sus semejantes, superando la necesidad de competir en el mundo de la subsistencia.

Acceder al consenso como institución implica descubrir y reconocer el alter, alguien que surge cuando el individuo "supera lo íntimo de sus pen- 
samientos, la formación de sus decisiones, de sus dudas que aún a veces no alcanzan a ser claramente formuladas; de aquello que reprime, o que aún no ha expresado o que quizás nunca expresará, no tanto porque no lo desee, sino porque es inexpresable" (Garzón, 2005).

Entonces, para que exista consenso se requiere reconocer al otro, implica definir y adoptar un espacio que sea a la vez "tierra de nadie y común a todos". Lugar en el que cesan ciertas posibilidades unilaterales y por tanto, se debe renunciar a ellas, ceder en algo, o de alguna forma permitir o aceptar al otro. Nace entonces un espacio que individualmente no pertenece a alguien en particular, pero que colectivamente es de todos: el espacio común, público.

Pero para llegar al consenso, es preciso establecer una red de necesidades comunes al grupo. En términos de lugar, su identificación sería factor motivante de la "polis" como espacio físico y social en el cual confluye la identificación de necesidades que dan lugar a relaciones de supervivencia. Dicho espacio incluye el dedicado al descanso y la reparación de energías y debe relacionarse con el de otros semejantes. De aquí, al pasar a otro tipo de consensos, que se van haciendo menos explícitos en la medida que crece el tamaño del grupo, influyen dos aspectos significativos: de un lado, la no interferencia mutua y del otro, el apoyo compartido frente a alguna amenaza externa común.

De la oposición y a la vez complemento entre lo privado y lo público según Lewin (op.cit), surge el entorno, como fenómeno vinculado al ambiente de la persona, el cual implica su interacción con aquel, tal como ella lo percibe subjetivamente: es su campo (Duarte, 2003). Esta subjetividad impone diferentes límites a la privacidad, que dependen del contexto cultural y social como ámbito donde imperan exclusivamente los deseos y preferencias individuales. En resumen, el reconocimiento del otro deviene en condición necesaria para el ejercicio de la libertad individual y por ende, fundamento del concepto de lo público.
En consecuencia, lo público representa el libre acceso de comportamientos y decisiones de los miembros dentro del grupo, que deben ser conocidos públicamente. En especial cuando las personas se destacan en el ejercicio de alguna función de preponderancia o liderazgo, como alguna posición con autoridad político-jurídica; en cuanto el individuo es más visible en el grupo, la transparencia de sus actos será clave para su aceptación y credibilidad dentro del mismo. Se convierte entonces la publicidad de sus actos, en elemento esencial del Estado de Derecho de forma tal que Kant la eleva a la categoría de principio trascendental (Santiago, 2004), pues sin ella "no habría justicia, que solo puede ser pensada como públicamente manifiesta, ni habría tampoco derecho, el cual solo se otorga desde la justicia".

Entonces, el concepto de espacio público puede ser generalizado como lugar donde lo heterogéneo de la sociedad se funde en una coexistencia pacífica y armoniosa, donde se conforma y se confirma la posibilidad de convivir sin atropellarse unos a otros (Berroeta, \& Rodríguez, 2010). Tal espacio, que evidencia lo que se permite en sociedad, es aquello en lo cual nos ponemos de acuerdo como un conjunto de propuestas mediante las cuales se superan las diferencias, sin relegar ni desconocer el ámbito privado. Por ende, el espacio público se identifica como el escenario para la libre expresión de la autonomía de las personas en tanto encuadren en él, aunque vivan una cierta separación de su vida privada.

En lenguaje político, la esfera pública es un constructo en el que cada ser humano se ve reconocido como tal en relación con los otros, con los que se vincula a partir de compromisos que se renuevan. Esto es, según Sahui (2000), citado por (Delgado, 2011) un "espacio de encuentro entre personas libres e iguales que razonan y argumentan en un proceso discursivo abierto dirigido al mutuo entendimiento y a su auto comprensión normativa". Es este el fundamento de racionalización democrática de la política, por el cual se puede concebir la ciudad como una asociación libremente locali- 
zada en una geografía dada, que implícitamente reúne seres humanos para la satisfacción de sus necesidades individuales y colectivas a través de la existencia de un espacio común; personas cuya finalidad no solamente es subsistir sino "vivir bien" en toda la acepción de la palabra, insertas en un lugar donde "vivir bien" no es necesariamente una solución de carácter material, sino un estatus que también satisface el campo de las relaciones: la capacidad de participar en las decisiones colectivas como expresión propia del carácter social del ser humano. En un sentido más general, es aquí donde lo colectivo toma conciencia del poder de asociación, plasmando como piedra angular, la sinergia resultante del auto reconocimiento y la consiguiente capacidad de ocupar un lugar (ego), con la de reconocer la existencia de otros semejantes (alter) para participar en la toma de decisiones comunes. (Proshansky, Fabián, \& Kaminoff, 1983).

El vivir bien para los filósofos atenienses Platón y Aristóteles, equivale a vivir virtuosamente, como la forma más apropiada a la naturaleza de cada uno, conforme con su esencia, es decir, tras la consecución del bien propio, o sea la excelencia, la entelequia, que en la filosofía aristotélica indica la perfección propia del acto, la cual se obtiene cuando se llega a su realización definitiva; es la meta del obrar, el punto de llegada de los dinamismos, la posesión de la perfección por parte de las cosas (Atencia., Gavilán., \& Rodríguez, 1993). Y lo anterior solo se logra en un espacio social en donde se pueda participar plenamente en la toma de decisiones. En términos actuales, esa participación podría concebirse como integración social, entendida como la comunicación en doble dirección, donde la interacción se da como participación del individuo en lo social y como contraparte, donde el entorno contribuya a la formación de pautas positivas del comportamiento, la personalidad y los valores. Desde este punto de vista, el espacio público se justifica por la necesidad de un lugar de deliberación acerca del destino social de la ciudad en conjunto, el cual no logrará "despegar" (si se acepta la metáfora), mientras los seres humanos continúen "pegados, adheridos" al espacio personal individual que cada cual requiere para sí (egoísmo).

2.4 Individuo, espacio urbano y su comportamiento en el tránsito urbano

En contravía con los postulados teóricos sobre la construcción del entorno urbano, que orientarían al avance hacia el logro de una "ciudad por el hombre y para el hombre", el lugar para vivir bien, el súmmum de civilidad, entre otros aspectos, en lo relacionado con la movilidad se convierte en preocupante el tema de la accidentalidad asociada al tráfico urbano, en correlación con tendencias de crecimiento sostenido especialmente en países de ingresos bajos y medios quienes, paradójicamente, concentran solo el $48 \%$ de los vehículos del mundo (Gutiérrez, et al, 2014), donde el ingrediente de comportamiento transgresor o riesgoso, como se diría en jerga de salubridad pública, resulta altamente resistente a su extinción. En la búsqueda de explicación a este fenómeno se ha comenzado a aplicar un modelo de Comportamiento Planificado (Ajzen, 1988), citado por (Martín, Martínez, \& Rojas, 2011), que sostiene que la conducta humana es voluntaria y está determinada por la intención conductual, la cual a su vez se construye a partir de tres procesos principales: actitudes sociales, norma subjetiva y control conductual percibido. Las actitudes sociales surgen de la interacción entre las expectativas conductuales y su valoración por parte de cada sujeto, en tanto que la norma subjetiva sería el modo en que el sujeto recibe e interpreta lo que dicen las personas y los grupos que considera relevantes acerca de lo que debería hacer en relación con la conducta y la motivación para acomodarse a estas opiniones, mientras que el control conductual percibido contiene las creencias que poseen los sujetos sobre su propia capacidad para realizar una conducta determinada.

Antes de avanzar en esta dirección, es conveniente aclarar el alcance y significado del término comportamiento y cómo este puede influir en una toma de posición en cuanto al fenómeno que se 
trata de dilucidar. Con demasiada frecuencia se repite un error semiológico consistente en el uso indiscriminado de los términos conducta y comportamiento para denominar la actuación de un sujeto. Pues a pesar de que actuación o realización del acto es parte de la conducta, también puede serlo el comportamiento; sin embargo, en la psicología y la psicotécnica las disciernen claramente, en cuanto "conducta" es un hecho condicionado por acciones o factores externos o internos, con la cual el sujeto proyecta lo que es, de manera espontánea como respuesta a determinados estímulos, el comportamiento es, más bien un hecho que se realiza con rigurosa intención y libre de todo condicionamiento. Por tal razón, se toma el comportamiento como expresión cultural, de acuerdo a como el individuo percibe y actúa en el espacio urbano.

Entonces, si el comportamiento se asocia como acción deliberada, es posible que las tendencias de crecimiento sostenido de transgresión de las normas comunes de tránsito por parte de los usuarios del espacio vial urbano, peatones y conductores, permite suponer que ellos establecen diferencias entre el hecho transgresor, ante el cual un observador lo perciba de tal forma que la sanción que este esperaría por la transgresión, le genere actitudes de rechazo, o de indiferencia, ante la cual, si la sanción correspondiente no es inmediata, o no se ejecuta, podría convertirse en refuerzo negativo y a su vez, ser asumida como comportamiento aceptable, $\mathrm{o}$ antivalor.

La trasgresión a la norma en el tránsito, cuando no genera un accidente físico o la percepción de un peligro inminente, a menudo puede generar algún sentimiento de recompensa inmediata en términos de incremento de la autoestima, el ahorro en energía gastada, en tiempo o en comodidad, induciendo a la formación de una escala de antivalores, o de reconocimiento a cierto grado de superioridad dentro de la manada por la acción osada, o que al menos, libra de consecuencias negativas inmediatas. Es lo que ocurre cuando una persona asume el liderazgo momentáneo de un grupo al tomar la iniciativa de cruzar una calle por donde, o en el instante que no se debe, se siente recompensado y se envanece al lograrlo. Tal es el caso de un padre, que llevando de la mano a sus hijos pequeños alcanza el objetivo de cruzar la calle, quien usualmente recrimina al más rezagado de su grupo, o a quienes titubearon por haber puesto en riesgo el éxito de su decisión o durante su ejecución. Vale anotar que mediante la aplicación del modelo de comportamiento, se han explicado (Muñoz, 2002) suposiciones que relacionan la actitud y el comportamiento en el tránsito, desde la percepción subjetiva del riesgo, o actitud de osadía como desafío a la norma, frente a la ignorancia o desconocimiento de la misma. Este aspecto presenta entonces, un promisorio filón para el estudio de relaciones relevantes, con el fin de buscar satisfactoriamente relaciones predictivas de riesgos predominantes en el tránsito urbano en situaciones específicas, como son: el género, el grado de escolaridad, el grupo etario, el grado de pertenencia y/o conocimiento del lugar, el tipo de ocupación o profesión, etc., con el fin de capacitar a los encargados de la administración y control del tránsito sobre un enfoque similar al que se realiza al tratar la epidemiología en el campo de la salubridad pública y la prevención, mediante la adopción de tratamientos específicos para cada caso, teniendo en cuenta estadísticas sobre el fenómeno creciente y sostenido de víctimas por accidentes de tránsito asociadas a un lugar específico.(Gutiérrez, op. cit.)

De otro lado, si se acepta el papel relevante de la motivación que lleva a circular en el espacio urbano, donde la urgencia es comúnmente uno de los factores clave para la elección del tiempo que la persona juzga adecuado invertir en su desplazamiento (Alcántara, 2010), resultaría de gran utilidad la prevención mediante la correcta priorización entre la provisión del equipamiento para el mismo, infraestructura, y pertinencia del sistema informativo del tránsito, o acentuar el efecto psicológico de vigilancia para verificar la obediencia a la norma por parte del usuario, o la elección de opor- 
tunidades, modo, sitio y espacio de aplicación de medidas preventivas o sancionatorias oportunas.

\section{El papel de la educación en la circulación ur- bana}

A menudo las entidades responsables del mantenimiento de estándares de seguridad en la ciudad, promueven campañas para la prevención y el aseguramiento de la calidad de la circulación en un entorno urbano. Estas se centran en simples enunciados, donde los aspectos de efectividad y prevención se reducen a ciertas acciones implementadas genéricamente como prevención, control y/o sanción. En la fase preventiva, alternando la prohibición con el estímulo, la primera a través de barreras físicas como la construcción de obstáculos para separar los flujos de tránsito en conflicto, los cuales se convierten en instrumentos de discriminación con desmedro de las condiciones del más débil. Un ejemplo es cuando los peatones se ven obligados a recorrer trayectorias exageradamente largas, o a desarrollar esfuerzos adicionales frente a los vehículos motorizados a los cuales no se les altera sus recorridos; otro ejemplo es la instalación de mallas o cercados aledaños a los pasos peatonales, las cuales por sí solas, demuestran objetivamente el grado de deshumanización de la ciudad, su origen y finalidad.

La segunda forma de buscar el aseguramiento de calidad del tráfico urbano se realiza mediante estímulos, apelando a la semiótica o ciencia del análisis del comportamiento de la mente, a partir de mensajes emitidos mediante diferentes modelos de significantes; estos consisten en símbolos reconocibles a los que se les asigna la función de advertir o recordar comportamientos específicos, con el fin de generar respuestas inmediatas en un sentido (Eco, 2000). A este tipo pertenece el sistema de señalización y demarcación vial, el cual a través de mensajes gráficos, o ideogramas sencillos o estímulos sonoros, reconocibles de un vistazo, le recuerdan al usuario sobre una forma de comportarse en el tránsito, actuando como orientación, información o cohibición, según la inten- ción de quien la coloca (Ministerio de Transporte, 2015).

Por ejemplo, si se transita por una carretera y se advierte un cartel amarillo con la imagen simplificada de un menor dibujado dentro, se interpreta como la cercanía a una zona escolar y por tanto, exige disminuir la velocidad y prestar más atención a la circulación y presencia de personas a los lados de la vía. Esa sería una correcta interpretación del signo gráfico. Comportamiento que se persigue con el signo: transmitir información precisa y concisa de forma que pueda ser asimilada por el cerebro de manera rápida para tomar, en consecuencia, decisiones al respecto.

El seguimiento y control del movimiento en la vía, usualmente se realiza por alguien o mediante algo que ejercita funciones de testigo, sea un agente de tránsito o algún dispositivo tecnológico de monitoreo, como cámaras de televisión donde se pueden registrar operaciones erráticas o radares que miden la velocidad vehicular (Arteaga, 2010). Sin embargo, pese al efecto disuasivo del instrumento, estos recursos a menudo no alcanzan a generar el efecto esperado.

Entre las medidas mencionadas vale la pena recalcar la importancia de la prevención de la acción, por su efecto de recordatorio y acción educativa, en relación especialmente con el papel que se les asigna como capacitación a los nuevos usuarios de la vía, peatones o conductores, quienes requieren una capacitación preliminar que los habilite para ingresar en el tráfico vehicular. En este punto no basta con el entrenamiento psicomotor para conducir un vehículo, si no se refuerzan la formación en valores y el aprendizaje significativo acerca del compromiso y responsabilidad social que supone el acto de participar en el flujo vehicular (Díaz-Barriga, \& Hernández, 2002).

Es decir, a pesar que se logre algún nivel de destreza en la práctica de conducir un vehículo, deben reforzarse didácticas sobre aspectos valorativos como el reconocimiento del otro, sus espacios, etc. 
lo cual no puede reducirse a la simple respuesta esquemática de cuestionarios donde se registren aciertos logrados por simple evocación y/o repetición de algunos apartes del Código de Tránsito, tal como la acción que en la actualidad se realiza por instructores que a menudo no cuentan con la debida capacitación pedagógica.

En cuanto a la capacitación que supone el ingreso del peatón como usuario de la vía, por supuesto, el tema es mucho más agudo y preocupante, pues, por antonomasia, el uso de la vía pública a pie no requiere autorización: la práctica social solamente establece que los miembros de grupos etarios extremos, como infantes y tercera edad, o las personas con cierto grado de capacidad física reducida: visión, audición o movilización, deban estar acompañados por otras personas. Así, a pesar de variadas y recientes disposiciones sobre convivencia urbanística en este sentido, el camino hacia la priorización y reconocimiento de sus derechos, se halla aún en una etapa incipiente.

\section{Conclusiones}

En primer lugar, aunque el enfoque disciplinar para el estudio del tránsito se ha basado en la interacción de tres factores esenciales, el usuario, el vehículo y la vía, donde el tema de la seguridad y la calidad del uso de la vía se aborda desde un punto de vista lineal que busca mejores resultados, a partir de la accidentalidad que ocurre, resulta altamente motivante intentar una mejor aproximación interdisciplinar al fenómeno de la movilidad urbana. Es interesante abordar la temática desde otros ángulos que enriquezcan y amplíen la gama de puntos de vista, en relación con el tema del comportamiento, desde los puntos de vista filosófico fundamentado en la necesidad del auto reconocimiento como base para descubrir al otro y base ontológica en la comprensión de conceptos sobre las connotaciones del espacio: vital, social, público, urbano, y de allí derivar la importancia de las motivaciones extrínsecas e intrínsecas del sujeto en el uso y apropiación de dichos espacios en relación con el uso de la vía y del modo utilizado para su desplazamiento, su justificación y motivos susceptibles de consensos o normas reguladoras.

En segundo lugar, se desprende la tremenda relevancia que tiene el enfoque holístico del concepto Espacio Público como base fundamental para la concreción de lo urbano, por las actividades que allí se construyen y transforman bajo dinámicas culturales y políticas que necesariamente deben revertir en prácticas educativas, su construcción y su transmisión para la formación en aspectos que entran en juego para mejorar la calidad de vida en el ámbito de la ciudad. Es este ámbito el escenario que puede servir de soporte a dicha refundación.

Por lo anterior, resulta cada vez de mayor relevancia insistir en la necesidad de intentar, o al menos proponer, la reorientación de la acción educativa en el ámbito urbano, en relación con la movilidad a partir de un enfoque del tema socio urbanístico donde se refuerce el significado de ciudad como constructo del hombre para el hombre y como espacio de inclusión, en el que la participación y la comunicación se sustenten bajo normas de moralidad, justicia y equidad, para lograr acuerdos en la búsqueda de una universalidad incluyente, sostenible y ecológica; en la que, ante los crecientes problemas de la movilidad urbana, se enfoquen programas de capacitación y sensibilización en estos temas con el fin de mejorar la percepción, actitud y sentido de responsabilidad en el uso del espacio urbano, por toda la sociedad.

Por tanto, es evidente que se trata de una tarea de todos el asumir la formación para la movilidad como parte de la capacitación ciudadana en el uso del espacio público urbano. Esta tarea no puede reducirse a unas cuantas asignaturas de currículo; al contrario, debe ofrecerse como conjuntos de experiencias didácticas generadas en actividades $y$ oportunidades variadas desde una apertura interdisciplinaria mediante la cual se relacionen contenidos con el mundo de los valores, la observación de problemas de índole social del entorno, bajo un ambiente de colaboración más activo entre estudiantes y profesor que potencien el desarrollo 
efectivo de vivencias de educación para la ciudadanía y el compromiso en la búsqueda de soluciones a los problemas generados en el uso del espacio público urbano.

\section{Referencias}

Alcántara, E. (2010). Análisis de la movilidad urbana. Espacio, medio ambiente y equidad. Bogotá, Colombia: CAF. Recuperado de: https://www.caf. com/

Arnoletto, E. (2007). Curso de Teoría Política. Recuperado de: http://www.eumed.net/

Arteaga, N. (2010). Video-vigilancia del espacio urbano: tránsito, seguridad y control social. Andamios. 7 (14), 263-286. Recuperado de: http://www. scielo.org.mx/

Atencia, J., Gavilán J., \& Rodríguez, A. (1993). Iniciación a la Historia de la Filosofía. Málaga, España: Agora.

Bollnow, O., \& d'Ors, V. (1969). Hombre y espacio. Barcelona: Labor.

Berroeta, H., \& Rodríguez, M. (2010). Una experiencia de participación comunitaria de regeneración del espacio público. Revista Electrónica de Psicología Política, 8 (22), 1-26. Recuperado de: http:// www.unsl.edu.ar/

Camargo, D. (2003). Lógicas de poder y regulación de espacios (capítulo 1). Bogotá, Colombia: Unibiblos.

Delgado, M., \& Malet, D. (2011). El espacio público como ideología. España: Los libros de la Catarata. Recuperado de: http://www.fepsu.es/

Díaz-Barriga, F., \& Hernández, G. (2002). Estrategias docentes para un aprendizaje significativo. Una interpretación constructivista. México: Mc Graw Hill.
Recuperado de: https://jeffreydiaz.files.wordpress. com.

Duarte, J. (2003). Ambientes de aprendizaje: una aproximación conceptual. Estudios pedagógicos, 11397 ,29. Recuperado de http://rieoei.org/ index.php

Eco, U. (2000). Tratado de Semiótica General. Barcelona, España: Lumen. Recuperado de: http://psikolibro.blogspot.com

Garzón, E. (2005). Lo íntimo, lo privado y lo público. Mexico: Instituto Federal de Acceso a la Información Pública (IFAI). Recuperado de: http://www. itszn.edu.mx/

Gutiérrez C., Romaní F., Wong-Chero P., \& Montenegro-Idrogo J. (2014) Perfil epidemiológico de la discapacidad por accidentes de tránsito en el Perú, 2012. Rev. Peruana de Medicina, Experimental y Salud Pública, 31 (2), 267-268. Recuperado de: http:// www.scielo.org.pe/

Hall, E. (1981). Beyond culture. New York, USA: Anchor Books. Recuperado de: https://monoskop.org.

Hall, E. (2003). La dimensión oculta. México: Siglo Veintiuno. Recuperado de: https://docs.google.com.

Iranzo, V. (2005). Filosofía de la ciencia e historia de la ciencia. Quaderns de filosofia i ciència, 35, 19-43. Recuperado de: http://www.uv.es/

Jirón, P., \& Iturra, L. (2015). Momentos móviles. Los lugares móviles y la nueva construcción del espacio público. Arquitecturas del Sur, 30 (39), 44-57. Recuperado de: https://www.researchgate.net/

Lewin, K. (1964). Field theory in social science: selected theoretical papers. Ed. Por Dorwin Cartwright. New York, USA: Harper and Row Recuperado de: http://psycnet.apa.org/ 
Martín, M., Martínez, J., \& Rojas, D. (2011). Teoría del comportamiento planificado y conducta sexual de riesgo en hombres homosexuales. Revista Panamericana de Salud Pública. 29 (6), 433-436 Recuperado de: http://www.scielosp.org/

Migueles, M. (2006). Fundamentación epistemológica del Enfoque Centrado en la Persona. Polis, Revista de la Universidad Bolivariana, 5 (15). Recuperado de: https://polis.revues.org/4914

Ministerio de Transporte. (2015). Manual de Señalización Vial. Dispositivos uniformes para la regulación del tránsito en calles, carreteras y ciclorutas. Recuperado de: https://www.medellin.gov.co

Muñoz, S. (2002). Factores Psicológicos que incrementan el riesgo de accidentes de circulación. $R e-$ vista Internacional de Psicología, 3 (02). Recuperado de: http://www.revistapsicologia.org/index. php/revista

Páez, D., \& Blanco, A. (2006). La teoría sociocultural y la psicología social actual. 27-61. Madrid, España: Fundación infancia y aprendizaje. Recuperado de: https://openaccess.leidenuniv.nl/
Popper, K. (1987). Realismo y el objetivo de la ciencia. Madrid, España: Técnos. Recuperado de: http://www.raularagon.com.ar/

Proshansky, H., Fabián A., \& Kaminoff, R. (1983). Place-identity: physical world socialization of the self. Journal of Environmental Psychology, (83 -57, (3. doi: 10.1016/S0272-4944(83)800218

Sanfeliu, I., \& Varona, J. (2010) Migración, racismo y poder. El narcisismo de las pequeñas diferencias. Madrid, España: Biblioteca Nueva. Recuperado de: http://www.imagoclinica.com/

Santiago, T. (2004). Kant y su proyecto de una paz perpetua (en el bicentenario de su muerte). Revista Digital Universitaria 5 (11) 4-5. Recuperado de: http://www.revista.unam.mx/

Tomadoni, C. (2007). A propósito de las nociones de espacio y territorio. Reflexión, 10 (4). Recuperado de: http://www.bdigital.unal.edu.co/

Wirth, L. (2005). El urbanismo como modo de vida. Bifurcaciones, (002), 1-15. Recuperado de: http://redalyc.claemex.mx 